\title{
Evidence of Delocalized Excitons in Amorphous Solids
}

\author{
Fabrizio Messina, Eleonora Vella, ${ }^{*}$ Marco Cannas, and Roberto Boscaino \\ Dipartimento di Scienze Fisiche ed Astronomiche, Università degli Studi di Palermo, Via Archirafi 36, I-90123 Palermo, Italy
} (Received 17 December 2009; published 7 September 2010)

\begin{abstract}
We studied the temperature dependence of the absorption coefficient of amorphous $\mathrm{SiO}_{2}$ in the range from 8 to $17.5 \mathrm{eV}$ obtained by Kramers-Kronig dispersion analysis of reflectivity spectra. We demonstrate the main excitonic resonance at $10.4 \mathrm{eV}$ to feature a close Lorentzian shape redshifting with increasing temperature. This provides a strong evidence of excitons being delocalized notwithstanding the structural disorder intrinsic to amorphous $\mathrm{SiO}_{2}$. Excitons turn out to be coupled to an average phonon mode of $83 \mathrm{meV}$ energy.
\end{abstract}

DOI: 10.1103/PhysRevLett.105.116401

PACS numbers: 71.35.Cc, 71.23.- $\mathrm{k}, 78.40 . \mathrm{Pg}$

Excitons are neutral quasiparticles consisting in an electron-hole pair bound via Coulomb interaction. One of the most important features of excitons is their mobility, providing a fundamental mechanism of site-to-site energy transfer in condensed matter without charge transport. While being one of the most complex manifestations of the electronic band structure of a solid, excitons play a key role in many processes occurring not only in solid state but also in nanoscale systems as well as in molecular materials. These include light emission in semiconductor devices, energy capture in photovoltaic systems [1] or generation of point defects upon exposure to high power laser light [2]. Furthermore, exciton dynamics may feature collective phenomena such as Bose-Einstein condensation which are currently the subject of intense research [3]. Exciton mobility in a crystal is influenced by their interaction with phonons, leading in some cases to the trapping of the quasiparticle. In disordered systems lacking translational order such as glasses, the very existence of excitons was disputed for some time, but it is widely accepted now. However, the properties of excitons in such systems are still debated [4], those concerning the influence of structural disorder on their mobility being poorly understood as yet. This is particularly true for wide band gap insulators, of which amorphous silicon dioxide $\left(a-\mathrm{SiO}_{2}\right)$ is an archetypal system, because their excitonic absorption is difficult to investigate experimentally, since this requires vacuumUV $(\lambda<200 \mathrm{~nm})$ optical technologies. $a-\mathrm{SiO}_{2}$ is a solid of primary technologic importance in optics and microelectronics. It has lately received renewed attention aiming to further improve its optical properties in view of prospective vacuum-UV applications [5,6]. A full comprehension of exciton properties needs the thorough understanding of the fundamental band-to-band transition, which is among the basic open problems in the physics of $a-\mathrm{SiO}_{2}[5,7]$. This study has often been aided by the comparison with $\alpha$-quartz $\left(c-\mathrm{SiO}_{2}\right)$, the most common crystalline polymorph of silicon dioxide. Several experimental results, such as the detection of photoconductivity starting from $\sim 9 \mathrm{eV}$ [8], led to estimate the latter value as the band gap of $a$ - and $c-\mathrm{SiO}_{2}$ [8-11]. Some studies questioned these findings, by reporting a marked increase of photoconductivity and photoemission signals only above $11 \mathrm{eV}$, accompanied by qualitative variations of the photoelectron energy distribution $[5,12]$. For this reason the actual band gap of $\mathrm{SiO}_{2}$ was proposed to lay around $11 \mathrm{eV}$ [12], but this is still a matter of debate. The experimental investigation of these issues in $\mathrm{SiO}_{2}$ requires the combined use of different techniques. Optical absorption spectroscopy can be applied from $\sim 8$ to $\sim 9 \mathrm{eV}$, where the absorption coefficient $\alpha$ is smaller than $10^{3}-10^{4} \mathrm{~cm}^{-1}$ and both $a$ - and $c-\mathrm{SiO}_{2}$ feature the exponential absorption region usually referred to as the Urbach tail [7]. Reflectance spectroscopy is necessary at higher energies, where $\alpha$ becomes exceedingly high for optical absorption so that absorption can be only indirectly inferred by Kramers-Kronig (KK) analysis of reflectivity data. KK analysis can be quite tricky due to the need of reliable measurements over an extended energy range. This has limited the number of $\mathrm{KK}$ investigations on $\mathrm{SiO}_{2}$ to date $[11,13,14]$ and consequently hindered the comprehensive understanding of near-edge absorption in wide band gap amorphous systems. The peak at $10.4 \mathrm{eV}$ in the absorption spectra of $a$ - and $c-\mathrm{SiO}_{2}[5,9,13,14]$ is widely accepted to be an excitonic transition, based on its temperature dependence observed in $c-\mathrm{SiO}_{2}$ from 300 to $600 \mathrm{~K}$ $[9,13]$. However, almost no experimental data exist on the temperature dependence of the $10.4 \mathrm{eV}$ peak below $300 \mathrm{~K}$, especially in $a-\mathrm{SiO}_{2}$. Most important, the shape of this resonance is currently an open issue. Well established theoretical models describe the broadening of an exciton resonance in a crystal as resulting from exciton-phonon coupling (EPC) and the excitonic line shape as arising from the competition between mobility of excitons and thermal fluctuations of their energy [15]. Although no models exist for amorphous solids, we argue that a similar description could be used for them as well since it was suggested that the effects of a small structural disorder in crystalline systems are indistinguishable from those of thermal fluctuations [15]. In this Letter we contribute to the clarification of these topics by providing experimental data on the 
temperature dependence of the reflectivity spectra of the model system $a-\mathrm{SiO}_{2}$ below $300 \mathrm{~K}$. Our data yield strong evidence of the existence of delocalized, closely crystallike, excitons in an amorphous system.

We measured reflectivity $R(E)$ for $9 \mathrm{eV}<E<21 \mathrm{eV}$ (wavelength accuracy $\pm 0.1 \mathrm{~nm}$ ) of commercial synthetic $a-\mathrm{SiO}_{2}$ and $z$-cut $c-\mathrm{SiO}_{2}$ samples, featuring industrial grade optical polishing. Measurements were carried out in ultrahigh vacuum in near-normal geometry under synchrotron radiation at beamline $\mathrm{I}$ in HASYLAB (DESY). The reflected beam was detected by a photomultiplier coated by $\mathrm{C}_{7} \mathrm{H}_{5} \mathrm{NaO}_{3}$. Each acquired spectrum was scaled for the spectral density curve of the excitation beam. Experimental spectra were extended on the low and high energy tails using literature data down to $0 \mathrm{eV} \mathrm{[13]} \mathrm{and} \mathrm{the} \mathrm{ordinary} \mathrm{analytical}$ form in the free-electron gas approximation [16], $R(E) \propto$ $E^{-4}$, for $E>21 \mathrm{eV}$. Finally, standard KK analysis was performed on $R(E)$ in order to obtain the absorption coefficient $\alpha(E)$. The influence on the $10.4 \mathrm{eV}$ peak of different reasonable choices for the high energy extrapolation was verified to be very poor. Figure 1 (a) shows the reflectivity spectra of $a-\mathrm{SiO}_{2}$ from 10 to $300 \mathrm{~K}$ and that of $c-\mathrm{SiO}_{2}$ at $10 \mathrm{~K}$ for comparison. In $a-\mathrm{SiO}_{2}\left(c-\mathrm{SiO}_{2}\right)$ spectra feature four main peaks: a first comparatively narrow and more intense peak at $\sim 10.3 \mathrm{eV}(\sim 10.4 \mathrm{eV})$ and three other broader peaks at $\sim 11.5(\sim 11.4 \mathrm{eV}), \sim 14.0(\sim 14.1 \mathrm{eV})$ and $\sim 17.3 \mathrm{eV}(\sim 17.3 \mathrm{eV})$, respectively. The positions of the peaks in $a$ - and $c-\mathrm{SiO}_{2}$ agree qualitatively with those reported in literature $[5,11,13,14]$. In $a-\mathrm{SiO}_{2}$ the peaks at $\sim 10.3$ and $\sim 11.5 \mathrm{eV}$ change in both their positions and amplitudes as a function of temperature, whereas the peaks at $\sim 14.0$ and $\sim 17.3 \mathrm{eV}$ increase their intensities without noticeably changing their shapes with increasing temperature. As visible in Fig. 1(b), with increasing temperature the peak at $\sim 10.3 \mathrm{eV}$ redshifts while its amplitude decreases. In Fig. 2(a) the absorption coefficient of $a-\mathrm{SiO}_{2}$ is presented as calculated by $\mathrm{KK}$ analysis on spectra in Fig. 1. Four main peaks are present as well: at $\sim 10.4$, at $\sim 11.6$, at $\sim 14.1$ and

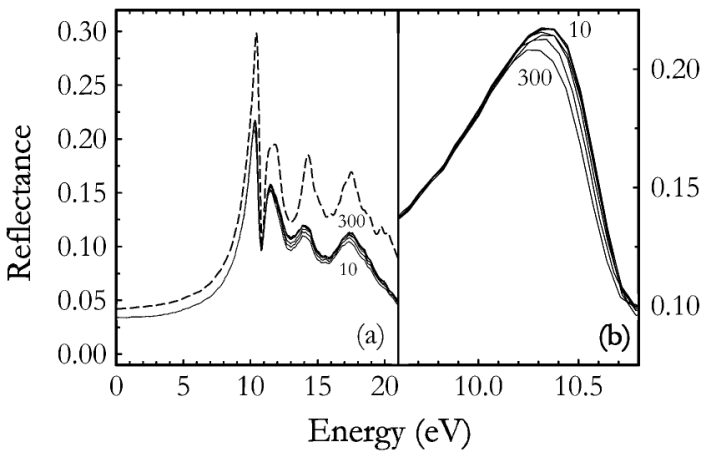

FIG. 1. (a) Reflectivity of $a-\mathrm{SiO}_{2}$ from 10 to $300 \mathrm{~K}$ by steps of $50 \mathrm{~K}$ (solid lines), and reflectivity of $c-\mathrm{SiO}_{2}$ at $10 \mathrm{~K}$ (dashed line). (b) Enlargement of the peak at $\sim 10.3 \mathrm{eV}$ in $a-\mathrm{SiO}_{2}$. Labels represent temperatures $(\mathrm{K})$ of the closest curve. at $\sim 17.3 \mathrm{eV}$. Figure 2(b) shows that the peak at $\sim 10.4 \mathrm{eV}$ redshifts with increasing temperature while its amplitude slightly decreases, moreover it is evident that in $c-\mathrm{SiO}_{2}$ the peak is narrower and more intense. Since the analysis of the peak at $\sim 10.4 \mathrm{eV}$ is the main purpose of the present work, the properties of the other peaks will not be discussed in the following. The peak at $\sim 10.4 \mathrm{eV}$ turns out to have a very good Lorentzian profile at all temperatures:

$$
\alpha(E, T)=\alpha_{0}\left[1+\left(\frac{E-\left[E_{0}^{0}+\Delta_{0}(T)\right]}{\Gamma_{0}(T)}\right)^{2}\right]^{-1}
$$

Figure 3 presents the normalized $\sim 10.4 \mathrm{eV}$ peak of $a-\mathrm{SiO}_{2}$ at 10 and $300 \mathrm{~K}$ and that of $c-\mathrm{SiO}_{2}$ at $10 \mathrm{~K}$, together with Lorentzian best fit curves: agreement with experimental data is very close even on the left band wings, as visible from the semilogarithmic plot in inset (b). We verified that introducing a baseline accounting for band-to-band absorption does not alter appreciably the Lorentzian line shape, only slightly decreasing the width. The position of the peak [inset (a)], as obtained by the Lorentzian fitting, is approximately constant around a value of $\sim 10.41 \mathrm{eV}$ from 10 to $150 \mathrm{~K}$, whereas it shifts to lower energies for higher temperatures. No change of the full width at half maximum (FWHM) of the peak is appreciable within the experimental error: its value is $(0.85 \pm 0.08) \mathrm{eV}$. In $c-\mathrm{SiO}_{2}$ at $10 \mathrm{~K}$ the peak is at $10.5 \mathrm{eV}$ and its FWHM is $(0.69 \pm 0.04) \mathrm{eV}$.

Although present data do not appear in contrast with previous ones [5,13,14], the accuracy of our measurements allows an unprecedented characterization of the $\sim 10.4 \mathrm{eV}$ peak, leading to unanticipated insights on the physics of excitons in $a-\mathrm{SiO}_{2}$. We demonstrated the excitonic peak at $\sim 10.4 \mathrm{eV}$ to have a remarkably good Lorentzian line shape at all the examined temperatures. The absorption line shape of excitons in a crystal is basically governed by the competition between their mobile nature and their tendency to localization in the lattice due to EPC. The former is measured by the half-width $B$ of the energy band of excitons, proportional to site-to-site transfer rate, while the latter is measured by the root mean square

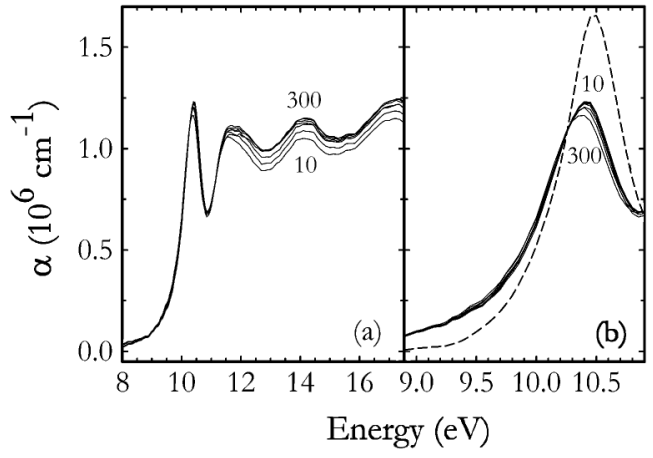

FIG. 2. (a) Absorption coefficient of $a-\mathrm{SiO}_{2}$ from 10 to $300 \mathrm{~K}$ by steps of $50 \mathrm{~K}$. (b) Enlargement of the peak at $\sim 10.4 \mathrm{eV}$ in $a-\mathrm{SiO}_{2}$ (solid lines) and in $c-\mathrm{SiO}_{2}$ (dashed line). Labels represent temperatures $(\mathrm{K})$ of the closest curve. 


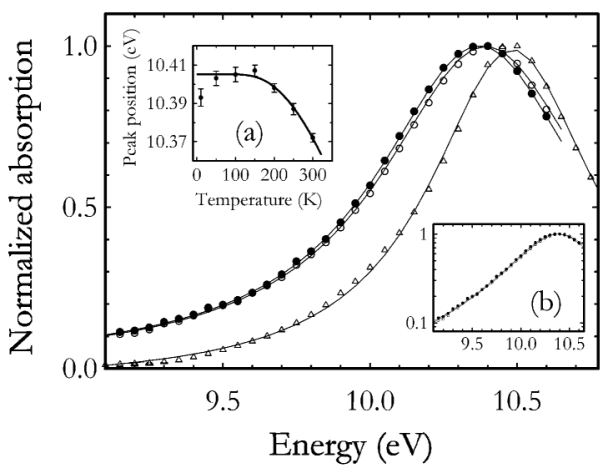

FIG. 3. Normalized absorption at $10 \mathrm{~K}$ (open circles) and at $300 \mathrm{~K}$ in $a-\mathrm{SiO}_{2}$ (filled circles), at $10 \mathrm{~K}$ in $c-\mathrm{SiO}_{2}$ (triangles). Continuous lines were obtained via a Lorentzian fit of each spectrum. (a) Position of the peak in $a-\mathrm{SiO}_{2}$ as a function of temperature (b) Semilogarithmic plot of the same data on $a-\mathrm{SiO}_{2}$ as in the main panel.

amplitude $D$ of the fluctuations of exciton energy due to the thermal activated vibrations of the lattice. Very general theoretical arguments (Kubo oscillator) [17] strongly suggest that Lorentzian absorption line shapes are expected when mobility effects $(B)$ prevail over energy fluctuations $(D)$. Indeed, the broadening of a resonance whose proper energy undergoes random Gaussian fluctuations of amplitude $D$ can be shown to be either Lorentzian or Gaussian when $D$ is, respectively, much smaller or larger than $\hbar / \tau$, where $\tau$ is the correlation time of the fluctuations. We physically expect the mean time $\hbar / B$ between site-to-site hops of excitons to basically represent $\tau$ (neglecting intersite correlations). Thus, the broadening is Lorentzian if $D \ll B$. A detailed model of exciton-phonon interaction in solids [15] was developed by Toyozawa, confirming the same conclusion: if the ratio $D / B \ll 1$ and the mobile nature of the exciton is thus prevailing (weak scattering limit), the exciton absorption line shape is Lorentzian. This can occur either if EPC is inefficient (small $D$ ) or if excitons are mobile enough (large $B$ ) to compensate for its effects. In this case, in Eq. (1) $E_{0}^{0}$ is the position of the unperturbed exciton line, while $\Delta_{0}(T)$ and $\Gamma_{0}(T)$ are the real and imaginary parts of the self-energy of the exciton in the phonon field, giving rise to a temperature dependent shift $\left[\Delta_{0}(T)\right]$ and broadening $\left[\Gamma_{0}(T)\right]$ of the exciton line. The FWHM $=2 \Gamma_{0}(T)$ is given by $c D^{2} / B$, where the predicted value of the constant $c$ depends on the detailed features of the exciton band [18]. The effect of increasing structural disorder (e.g., dislocations, vacancies, interstitial atoms or impurities) can be argued to be qualitatively the same as that of increasing temperature, in that both lead to an increase of $D$. For thermal or structural disorder so large or for $B$ so small that $D / B \gg 1$ (strong scattering limit), the model foresees the overall exciton line shape to be Gaussian. While Lorentzian exciton line shapes have been reported in literature for several crystalline systems at low temperatures [19], our analysis shows that Toyozawa's model can be extended to an amorphous system. Present data clearly point out that from 10 to $300 \mathrm{~K}$, exciton mobility is so large as compared to the effects of both static disorder and EPC in $a-\mathrm{SiO}_{2}$ that the exciton band keeps its Lorentzian profile, not presenting any tendencies towards a Gaussian one even at the highest considered temperature $(300 \mathrm{~K})$. This result is remarkable because exciton mobility is ultimately related to the translational symmetry of the lattice. Thus, the lack of long range order typical of an amorphous solid turns out to have a negligible effect on the properties of excitons, meaning that the effect of static disorder in $a-\mathrm{SiO}_{2}$ is low enough to closely preserve crystal-like delocalization of excitons. Surprisingly enough, it appears that, at least at low temperature, the difference between the excitonic properties of $a$ - and $c-\mathrm{SiO}_{2}$ comes down to a greater width of the first exciton absorption peak in the amorphous system, $(0.85 \pm$ $0.08) \mathrm{eV}$ instead than $(0.69 \pm 0.04) \mathrm{eV}$, likely due to structural disorder introducing small additional site-tosite fluctuations of the exciton energy contributing to $D$. Furthermore, present results are completely inconsistent with the model widely accepted in $\mathrm{SiO}_{2}$ literature claiming a strong localization and consequently a low mobility of excitons in $a-\mathrm{SiO}_{2}[5,12]$. The evidence that the peak is accurately described by a simple Lorenztian function seriously casts doubt on its previously proposed decomposition into at least four Gaussian subbands ascribed to several types of localized excitons [5,12]. Finally we note that the behavior of $\mathrm{SiO}_{2}$ is deeply different from that of other systems such as $\mathrm{Si}$, where a dramatic change of the shape and broadening of the absorption peak at around $3.5 \mathrm{eV}$ are observed when going from the crystal to the amorphous solid [20].

Toyozawa's model predicts the position of the excitonic peak to depend on temperature according to: $E_{0}(T)=$ $E_{0}^{0}+\Delta_{0}^{0} \operatorname{coth}\left(\hbar \omega_{0} / 2 k_{B} T\right)$, where the parameter $\Delta_{0}^{0}$ represents the effect of the zero-point vibrations on the shift of the peak and $\hbar \omega_{0}$ is the energy of thermal vibrations described using Einstein's model. The effect of possible structural disorder is included in $E_{0}^{0}$. As evident in Fig. 3(a), this equation well describes the observed trend with $E_{0}^{0}=(10.79 \pm 0.05) \mathrm{eV}, \Delta_{0}^{0}=(-0.38 \pm 0.05) \mathrm{eV}$ and $\hbar \omega_{0}=(0.083 \pm 0.002) \mathrm{eV}$, with the only possible exception of the point at $10 \mathrm{~K}$ [21]. According to Toyozawa's theory, the width should have the same temperature dependence as the peak position, i.e., governed by $\operatorname{coth}\left(\hbar \omega_{0} / 2 k_{B} T\right)$. Under this assumption and using for $\hbar \omega_{0}$ the value reported above, the expected variation of the width from 10 to $300 \mathrm{~K}$ is $8 \%$, corresponding to $0.07 \mathrm{eV}$, and thus not detectable within our $0.08 \mathrm{eV}$ uncertainty on the FWHM. On the other side, we believe the observed decrease of the amplitude of the peak (Fig. 2), measurable with a better accuracy than the width, to be an indirect evidence of the increase of the width of the line itself. 
The fact that $E_{0}^{0}$ is not coincident with the converging point of the Urbach tails at different temperatures in both $a$ - and $c-\mathrm{SiO}_{2}\left(E_{U}^{0} \sim 8.7 \mathrm{eV}\right.$ and $\sim 9.1 \mathrm{eV}$, respectively [5]) indicates $\mathrm{SiO}_{2}$ to be an indirect band gap $(\sim 9 \mathrm{eV})$ material, featuring a direct exciton at $\sim 10.4 \mathrm{eV}$, as previously proposed by several authors [10]. Furthermore, $E_{0}^{0}-E_{U}^{0}$ provides a rough estimation of $B \sim 2 \mathrm{eV}$, whose value allows us to compare the above mentioned theoretical expression for the FWHM with our data and thus to estimate $D_{a} \sim 0.74 \mathrm{eV}$ in $a-\mathrm{SiO}_{2}$ and $D_{c} \sim 0.66 \mathrm{eV}$ in $c-\mathrm{SiO}_{2}$. Fluctuations of the exciton energy of such an order of magnitude imply a rather efficient coupling of excitons with vibrational modes, being the weak scattering condition anyway ensured by the large extension of the excitonic band, while the influence of structural disorder $\left(D_{a} \sim D_{c}\right)$ is very poor, as anticipated. Thus, $\mathrm{SiO}_{2}$ turns out to belong to that class of materials referred to as featuring a strong EPC but a weak scattering [15]. A similar behavior can be observed in some very wide band gap crystals such as LiF and $\mathrm{NaF}$, having relatively wide (FWHM $\sim 0.4 \mathrm{eV}$ in $\mathrm{LiF}$ ) yet Lorentzian exciton line shapes [19] and, as $\mathrm{SiO}_{2}$, all giving rise to self-trapped exciton luminescence.

In conclusion, the observed behavior of above-edge absorption as obtained by KK dispersion analysis of the reflectance spectra of $a-\mathrm{SiO}_{2}$ from 10 to $300 \mathrm{~K}$ demonstrates the applicability of Toyozawa's theory for excitonic line shapes to an amorphous system. The remarkably good and temperature independent Lorentzian profile of the main excitonic peak at $\sim 10.4 \mathrm{eV}$ proves that excitons in $a-\mathrm{SiO}_{2}$ are highly mobile and retain their delocalized nature, albeit both the disorder peculiar of the amorphous structure and a strong EPC. The average vibrational energy of phonons interacting with excitons $\left(\hbar \omega_{0}=83 \mathrm{meV}\right)$ was determined.

We acknowledge financial support received from DESY and we thank the LAMP group for stimulating discussions.

*eleonora.vella@fisica.unipa.it

[1] S. Koizumi et al., Science 292, 1899 (2001); Y. Taniyasu, M. Kasu, and T. Makimoto, Nature (London) 441, 325
(2006); G. D. Scholes and G. Rumbles, Nature Mater. 5, 683 (2006).

[2] A. L. Shluger and K. Tanimura, Phys. Rev. B 61, 5392 (2000); N. Fukata et al., Appl. Phys. Lett. 83, 3495 (2003); S. Ismail-Beigi and S. G. Louie, Phys. Rev. Lett. 95, 156401 (2005).

[3] H. Deng et al., Science 298, 199 (2002); J. Kasprzak et al., Nature (London) 443, 409 (2006).

[4] S. M. Vlaming, V. A. Malyshev, and J. Knoester, Phys. Rev. B 79, 205121 (2009); A. Huijser et al., J. Am. Chem. Soc. 130, 12496 (2008).

[5] Defects in $\mathrm{SiO}_{2}$ and Related Dielectrics: Science and Technology edited by G. Pacchioni, L. Skuja, and D. L. Griscom (Kluwer Academic Publishers, USA, 2000), ISBN 0-7923-6685-9.

[6] L. Skuja et al., Proc. SPIE 5122, 1 (2003); K. Kajihara, J. Ceram. Soc. Jpn. 115, 85 (2007).

[7] E. Vella and R. Boscaino, Phys. Rev. B 79, 085204 (2009).

[8] Z. A. Weinberg, G. W. Rubloff, and E. Bassous, Phys. Rev. B 19, 3107 (1979); T. H. DiStefano and D.E. Eastman, Solid State Commun. 9, 2259 (1971).

[9] K. Platzöder, Phys. Status Solidi 29, K63 (1968).

[10] C. Itoh et al., Phys. Rev. B 39, 11183 (1989); J. R. Chelikowsky and M. Schlüter, Phys. Rev. B 15, 4020 (1977).

[11] C. Bosio and W. Czaja, Europhys. Lett. 24, 197 (1993).

[12] A. N. Trukhin, J. Non-Cryst. Solids 149, 32 (1992).

[13] H. R. Philipp, J. Phys. Chem. Solids 32, 1935 (1971).

[14] G. L. Tan et al., Phys. Rev. B 72, 205117 (2005).

[15] M. Schreiber and Y. Toyozawa, J. Phys. Soc. Jpn. 51, 1528 (1982); Y. Toyozawa, Pure Appl. Chem. 69, 1171 (1997); Optical Processes in Solids (Cambridge University Press, Cambridge, England, 2003), ISBN 0-521-55605-8.

[16] D. J. Jones et al., J. Mater. Res. 14, 4337 (1999).

[17] R. Kubo, J. Math. Phys. (N.Y.) 4, 174 (1963).

[18] The first-order value of the constant is: $c \sim \pi$ [15].

[19] M. Piacentini, Solid State Commun. 17, 697 (1975); R. Sano, J. Phys. Soc. Jpn. 27, 695 (1969).

[20] D. E. Aspnes, A. A. Studna, and E. Kinsbron, Phys. Rev. B 29, 768 (1984).

[21] Admittedly, present results do not allow us to establish if the observed scatter of this datum points out a low temperature deviation from Toyozawa's theory as far as the temperature dependence of the peak is concerned. 